\title{
Actualités en cardiologie isotopique : applications des nouvelles caméras $\mathrm{CZT}^{2 / 2}$
}

\author{
Highlights in nuclear cardiology: Applications of new CZT cameras \\ F. Ben Bouallègue *, E. D'estanque, D. Mariano-Goulart \\ Service de médecine nucléaire, CHU Lapeyronie, 371, avenue du Doyen-Gaston-Giraud, 34295 Montpellier cedex 5, France
}

\begin{abstract}
Résumé
Nous proposons ici une synthèse succincte des avancées récentes en termes d'instrumentation des gamma-caméras orientées cardiologie. Y sont détaillées, en particulier, les possibilités offertes en imagerie de perfusion myocardique classique et en tomographie cavitaire. Y sont, par ailleurs, rapportés les résultats préliminaires relatifs à deux thématiques de recherche actives au CHU de Montpellier portant sur la quantification de réserve de perfusion myocardique par SPECT dynamique et sur la détection de zones gâchettes en post-SCA précoce par protocole double isotope ${ }^{201} \mathrm{Tl} /{ }^{123} \mathrm{I}-\mathrm{MIBG}$.
\end{abstract}

Mots clés : Cardiologie isotopique ; Caméra à semi-conducteur CZT ; Perfusion myocardique ; SPECT dynamique ; Double isotope

\begin{abstract}
We offer a brief overview of recent advances in the instrumentation of dedicated cardiac gamma cameras. We focus on the expected benefits in the fields of classical myocardial perfusion imaging and gated blood-pool SPECT. We also report some preliminary results regarding two active research topics at Montpellier University Hospital: quantification of myocardial perfusion reserve using dynamic SPECT, and detection of trigger zones after myocardial infraction using a ${ }^{201} \mathrm{Tl} /{ }^{123} \mathrm{I}-\mathrm{MIBG}$ dual isotope protocol.
\end{abstract}

Keywords: Nuclear cardiology; CZT camera; Myocardial perfusion; Dynamic SPECT; Double isotope

\section{Introduction}

En 2015, la cardiologie isotopique représente une part essentielle de l'activité quotidienne de tout médecin nucléaire. Grâce à son caractère intrinsèquement non invasif et à sa large disponibilité, elle a su depuis plusieurs dizaines d'années s'imposer dans l'éventail des procédures de cardiologie diagnostique. Elle a pour cela bénéficié du développement

\footnotetext{
Présentation faite lors des hivernales 2015, 25-29 janvier 2015.

* Auteur correspondant.

Adresse e-mail : faybenb@hotmail.com (F. Ben Bouallègue).
}

soutenu d'une technologie de pointe tant sur le plan de l'appareillage que sur celui des solutions logicielles [1-3]. En particulier, l'imagerie de perfusion myocardique apparaît comme l'outil idéal au dépistage, à la caractérisation et au suivi de la pathologie coronarienne, enjeu de santé publique à l'échelle planétaire.

En dépit d'une place croissante de l'imagerie TEP, la SPECT cardiaque au moyen de traceurs technétiés ou iodés (voire au ${ }^{201} \mathrm{Tl}$ ) reste la modalité la plus consensuelle et la plus accessible à l'heure actuelle. Les examens de routine fournissent une information fonctionnelle pertinente du point de vue diagnostique et pronostique sur différents paramètres fonctionnels : perfusion et viabilité, cinétique, innervation et risque rythmique. 
L'évolution majeure de ces dernières années dans le domaine de la SPECT cardiaque reste sans conteste la mise au point et la commercialisation de caméras à semi-conducteur dédiées aux explorations cardiologiques [4]. Les performances de ces dernières en termes de sensibilité et de résolution vont vraisemblablement modifier profondément à court et moyen terme l'activité de routine clinique. L'optimisation des protocoles d'acquisition devrait permettre d'accroitre le confort des patients et de réduire les doses reçues tout en satisfaisant à des exigences évidentes de rentabilité. Enfin, l'amélioration très significative de la capacité de ces nouvelles caméras à réaliser de l'imagerie dynamique ou en double isotope, tant vantée par les industriels, reste à valider en routine hospitalière mais offre néanmoins de réelles perspectives sur le plan de la recherche.

\section{Caméras d'Anger et à semi-conducteur}

Contrairement à la gamma caméra classique d'Anger constituée d'une ou plusieurs têtes mobiles, à collimation le plus souvent parallèle, et embrassant un large champ de vue en tournant autour du patient [5], les nouvelles caméras à semiconducteur sont composées d'une série de détecteurs individuels indépendants (10 à 20 selon les modèles) qui peuvent être fixes (Discovery NM530c de General Electrics) ou mobiles (data spectrum [D-SPECT]), à collimation sténopée (NM530c) ou parallèle (D-SPECT), et focalisés sur un volume d'intérêt restreint (la NM530c par exemple, se concentre sur une sphère d'environ $18 \mathrm{~cm}$ de diamètre autour du cœur du patient). Chaque détecteur individuel est constitué d'une matrice carrée

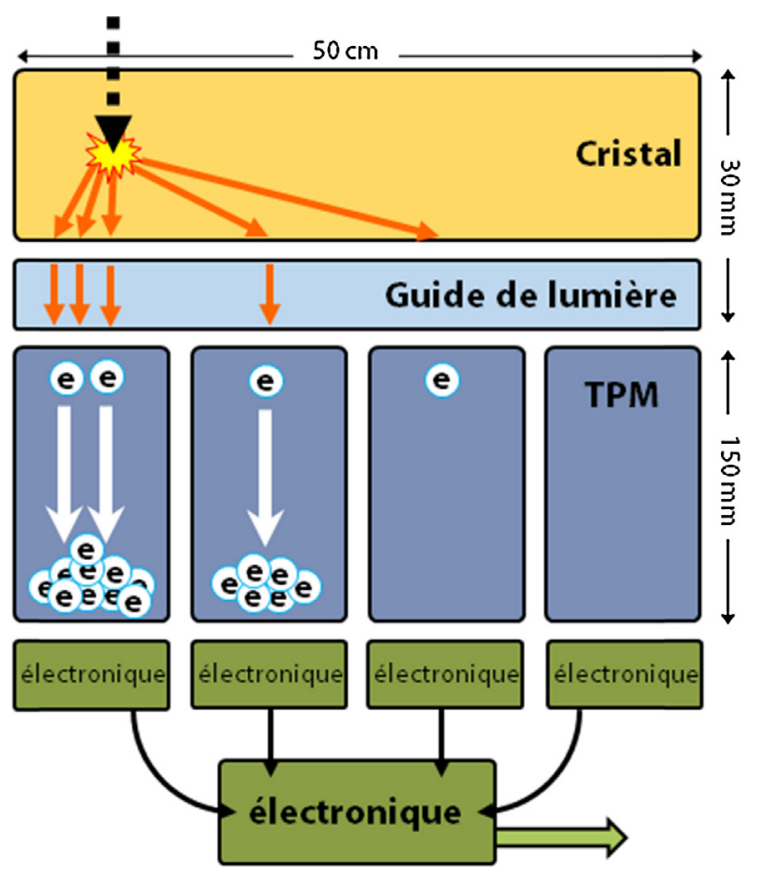

Fig. 1. Schéma général de détection d'une gamma caméra d'Anger. TPM : tube photomultiplicateur. Les dimensions caractéristiques sont indiquées. General detection scheme of a classical Anger gamma-camera. TPM: photomultiplier tube. The characteristic dimensions are indicated.

Adapté de Alcyone technology white paper - GE healthcare. de détecteurs élémentaires de taille millimétrique $(16 \times 16$ et 2,46 mm par exemple pour le module Imarad) qui sont autant de jonctions PN polarisées en inverse à base d'un alliage semiconducteur CdZnTe [6].

La physique de détection des rayonnements $\mathrm{X}$ et $\gamma$ dans le système classique d'Anger (Fig. 1) associe la création de photons visibles (ou proche UV) par luminescence au sein d'un cristal scintillateur (généralement du iodure de sodium dopé au $\mathrm{Tl})$, le transport de ces photons par un guide de lumière, puis leur interaction avec une photocathode donnant lieu à l'extraction de photo-électrons. Le rendement de détection est modéré, de l'ordre d'une dizaine de photo-électrons par keV d'énergie dans le rayonnement incident. Ces photo-électrons sont ensuite accélérés et amplifiés par une série de dynodes au sein d'un tube photo-multiplicateur (TPM) afin de générer un courant mesurable par un circuit électronique. La mesure des courants en sortie d'une batterie de TPM permet de localiser le rayonnement incident et d'en estimer l'énergie. La résolution généralement obtenue est de l'ordre de $4 \mathrm{~mm}$ en termes de localisation (résolution spatiale intrinsèque) et d'environ $10 \%$ pour le versant spectrométrique.

Un détecteur CZT détecte les rayonnements incidents par interaction directe au sein de la jonction $\mathrm{PN}$ avec création de paires de charges électron-trou (Fig. 2). Le rendement de détection est nettement amélioré, de l'ordre de 200 à 300 porteurs de charge par $\mathrm{KeV}$. Les électrons sont accélérés par une différence de potentiel et collectés via une anode dans un circuit intégré dédié. La résolution spatiale intrinsèque est ici limitée

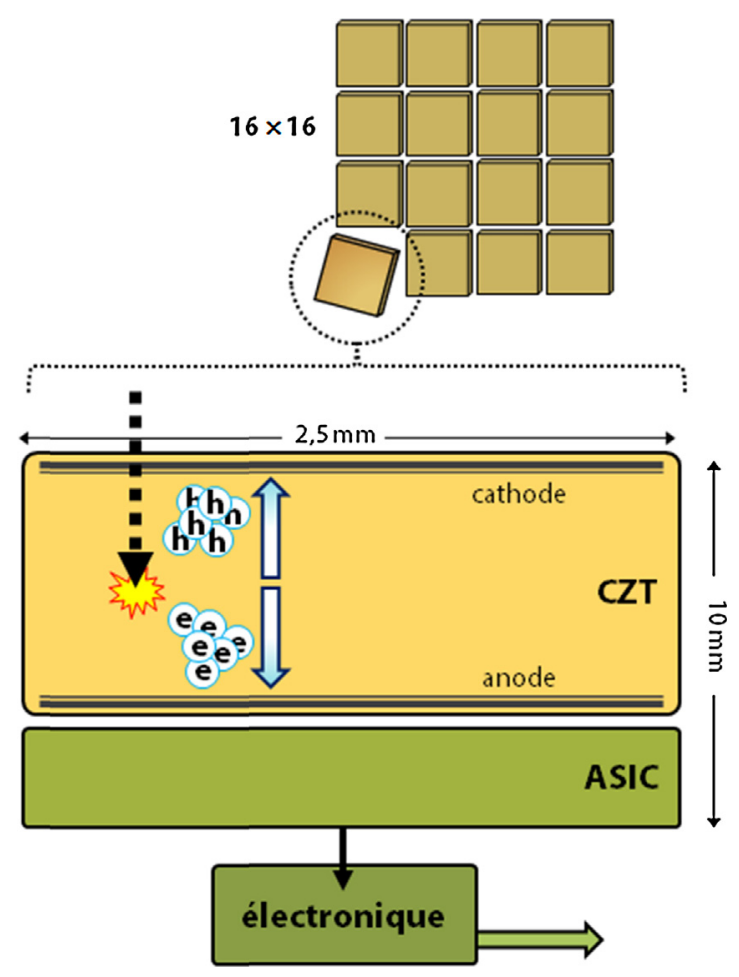

Fig. 2. Schéma de principe d'un détecteur élémentaire de caméra à CZT ASIC : circuit intégré dédié. Les dimensions caractéristiques sont indiquées. Schematic diagram of an elementary CZT detector. ASIC: application-specific integrated circuit. The characteristic dimensions are indicated. Adapté de Alcyone technology white paper - GE healthcare. 
théoriquement par les dimensions du nuage électronique (quelques centaines de $\mu \mathrm{m}$ ) et plus pragmatiquement par les dimensions du détecteur élémentaire (typiquement 2 à $3 \mathrm{~mm}$ ) dictées par des considérations techniques et financières. Du fait d'une très bonne statistique de comptage (30 000 porteurs de charge pour un photon de $140 \mathrm{KeV}$ ) associée à un bon rapport signal à bruit du signal électronique (facteur de Fano $<<1$ ), la résolution théorique en énergie est inférieure à $0,5 \%$. En pratique, en raison de différents facteurs dégradants (piégeage des électrons, bruit thermique), la résolution énergétique est de l'ordre de 2 à $5 \%$. Enfin, du fait d'une collecte individualisée du courant pour chaque jonction PN permettant de réduire le temps mort et d'éviter les phénomènes de saturation, la réponse $\mathrm{du}$ détecteur reste linéaire dans une très large gamme de statistique de comptage.

D'un point de vue pratique et comparativement aux potentialités de la gamma caméra classique, le gain en sensibilité d'un facteur 5 à 10 permis par les détecteurs CZT se traduit par une réduction des temps d'acquisition d'un facteur équivalent, ou alternativement d'une diminution combinée du temps de pose et de la dose injectée [7,8]. D'autre part, l'enregistrement des évènements en mode liste rend possible l'acquisition et la reconstruction d'images dynamiques sur de courtes durées de l'ordre de quelques secondes. Enfin, l'amélioration sensible des performances spectrométriques permet d'envisager la réalisation d'acquisitions en double-isotope.

\section{Imagerie de perfusion}

\subsection{Protocole rapide}

La tomoscintigraphie de perfusion myocardique occupe une place important parmi les procédures non invasives permettant la caractérisation et le suivi de la maladie coronarienne [9]. Elle permet avec une grande sensibilité d'orienter les patients à risque cardiovasculaire vers un geste de revascularisation en détectant des lésions ischémiques avant que celles-ci n'entraînent un retentissement fonctionnel ou des manifestations cliniques $[10,11]$. Elle peut aussi s'avérer utile à l'estimation de la viabilité de lésions constituées via la mesure de la redistribution tardive du thallium [12], bien que la TEP au ${ }^{18}$ FDG tende à s'imposer comme le gold standard en la matière [13].
La littérature de ces dernières années a clairement mis en évidence la supériorité des examens SPECT réalisés au moyen des nouvelles caméras à semi-conducteur par rapport aux gamma-caméra d'Anger en termes de qualité d'image, de capacité de détection des lésions ischémiques, d'estimation des paramètres fonctionnels, voire même de corrélation avec des mesures invasives de réserve de flux coronaire [14-20]. Audelà de ces performances techniques, l'avènement de la nouvelle génération d'appareils d'imagerie offre une réelle opportunité d'optimiser les protocoles d'acquisitions dans un souci de confort du patient et de réduction des doses administrées [21]. Prenant en compte les recommandations en vigueur, les spécificités de l'imageur exploité (GE Discovery NM530c) et les données de la littérature récente, un protocole rapide d'imagerie de perfusion a été mis en place au CHU de Montpellier (Fig. 3). Le protocole est réalisé sur un jour (sauf pour les patients obèses) et inclut de manière systématique et séquentielle une acquisition de repos après injection de $5 \mathrm{mCi}$ de ${ }^{99 m}$ Tc-tetrofosmine, une épreuve de stress maximale (physique et pharmacologique en l'absence de contre-indication) sous surveillance cardiologique, puis une acquisition d'effort après injection à l'acmé de l'effort de $15 \mathrm{mCi}$ de ${ }^{99 \mathrm{~m}} \mathrm{Tc}$ tetrofosmine. Les deux acquisitions durent 5 minutes et sont réalisées de manière synchronisée à l'électrocardiogramme. L'ensemble de l'examen requiert donc idéalement 30 minutes et, en pratique quotidienne, la durée totale fluctue entre 30 et 60 minutes. On se convaincra aisément que ce schéma d'acquisition permet naturellement d'optimiser l'occupation de la caméra puisque pendant l'épreuve de stress d'un patient donné seront réalisées l'acquisition d'effort du patient précédent et l'acquisition de repos du patient suivant. Une telle organisation en routine hospitalière permet de réaliser 8 à 10 scintigraphies myocardiques d'effort (et autant de repos) en une demi-journée.

Deux études récentes $[22,23]$ ont démontré la faisabilité d'un protocole d'imagerie de perfusion ultrarapide sur caméra CZT. Dans le cadre d'un protocole sur un jour, le repos suivant l'effort de 90 minutes, et un rapport de 3 entre les deux doses injectées, les acquisitions réalisées sur caméra à CZT durant 3 minutes aboutissaient à des résultats globaux et segmentaires intimement corrélés à ceux obtenus au moyen d'acquisitions de 15 minutes sur gamma-caméra d'Anger. Le protocole employé au CHU de Montpellier diffère en cela que l'enregistrement d'effort succède à celui de repos, pouvant faire craindre une
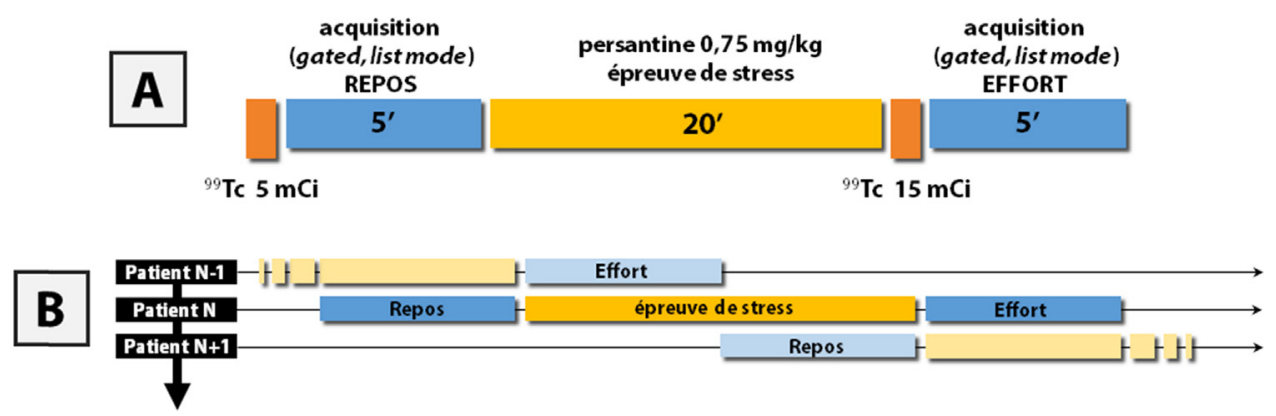

Fig. 3. Protocole de perfusion myocardique en 30 minutes. A. Schéma d'injection et d'acquisition. B. Schéma d'occupation de la caméra. A myocardial perfusion protocol in 30 minutes. A. Injection and acquisition scheme. B. Occupation of the camera. 
baisse de sensibilité liée à une altération par l'activité de repos résiduelle du contraste dans les images à l'effort. En réalité, considérant une demi-vie du traceur de 6 heures, un rapport de 3 entre les doses injectées à l'effort et au repos, un délai de 30 minutes entre les deux acquisitions, et postulant une extraction doublée en cas d'effort maximal, l'activité de repos parasite représente à peine $13 \%$ du signal présent dans l'image d'effort. L'expérience de routine clinique des mois écoulés et la confrontation au suivi clinique des patients et aux données angiographiques lorsqu'elles sont disponibles ont confirmé qu'il n'existe pas de baisse significative de précision diagnostique liée au protocole rapide employé. Enfin, la réalisation de l'acquisition d'effort après celle de repos permet de s'affranchir des stigmates d'une éventuelle sidération d'effort qui peut s'observer sur les images de repos lorsque celles-ci sont obtenues moins de 3 heures après le stress [24].

\subsection{Réserve de perfusion myocardique}

En scintigraphie de perfusion, on sait que l'interprétation qualitative classique en termes de defect et de réversibilité, en dépit de son excellente sensibilité, est parfois prise en défaut en particulier chez les patients pluri-tronculaires. Chez ces derniers, seule la sténose la plus sévère (du point de vue de l'ischémie d'aval) sera démasquée. À la limite, une pathologie tri-tronculaire équilibrée qui n'est pas systématiquement repérée par les données fonctionnelles (résultats de l'épreuve d'effort, analyse des données synchronisées à l'ECG, signes scintigraphiques indirects d'hypoperfusion globale telle que la visibilité du ventricule droit) peut être responsable d'un faux négatif.

Il existe de ce fait une réelle motivation pour développer une interprétation quantitative de la scintigraphie de perfusion en termes de débit (en $\mathrm{mL} / \mathrm{min} / \mathrm{g}$ ) et/ou de réserve de perfusion myocardique [25]. Cette analyse quantitative a fait ses preuves en TEP depuis une dizaine d'années, d'abord au moyen d'eau ou d'ammoniaque marquée puis de ${ }^{82} \mathrm{Rb}$, avec un intérêt à la fois diagnostique [26] et pronostique [27]. Des études récentes ont d'ores et déjà montré la faisabilité de telles mesures quantitatives en SPECT à la fois sur caméras à semi-conducteur [28] et sur gamma-caméra d'Anger [29] avec des résultats tout à fait convaincants bien que sur de petites cohortes et sans suivi prospectif.

D'un point de vue pharmacocinétique (Fig. 4), il existe une relation exprimée par une équation différentielle entre la concentration de radio-traceur dans le secteur circulant $\mathrm{V}$ et celle dans le myocyte $\mathrm{M}$. Cette relation est paramétrée par un ensemble de constantes d'échange modélisant la captation $\left(\mathrm{K}_{1}\right)$ et le lavage $\left(\mathrm{K}_{2}\right)$ du radio-pharmaceutique, voire son stockage dans les mitochondries $\left(\mathrm{K}_{3}\right)$ pour les cations lipophiles comme le MIBI ou la tétrofosmine. En particulier, la constante $\mathrm{K}_{1}$ est directement liée au débit de perfusion myocardique via l'équation de Renkin-Crone [30]. Dans ce contexte, les caméras CZT sont un outil idéal pour réaliser de l'imagerie dynamique afin d'extraire, après segmentation du myocarde et définition d'une ROI vasculaire, des courbes temps-activité (CTA) décrivant l'évolution des concentrations dans les

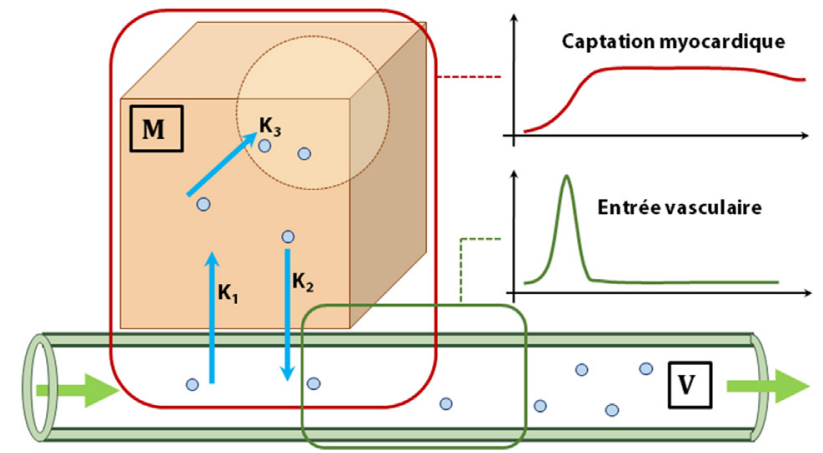

Fig. 4. Représentation schématique des échanges de radio-traceur entre le secteur circulant et les myocytes.

Schematic representation of tracer exchanges between vascular space and myocardial cells.

compartiments d'intérêt (entrée vasculaire et captation myocardique) fournissant, après résolution du modèle pharmacocinétique, les paramètres du modèle et in fine le débit de perfusion myocardique. La réserve de perfusion est définie comme le rapport des débits myocardique à vasodilatation maximale (après stress pharmacologique) et au repos. Elle traduit la capacité de la macro- et microcirculation myocardique à assurer une hyperhémie en cas d'accroissement des besoins métaboliques [31]. Son altération reflète de manière quantitative le degré d'ischémie myocardique avec un seuil de normalité fixé de manière consensuelle entre 2 et 2,5.

Un outil de traitement dédié a été développé au CHU de Montpellier et une étude de validation clinique a été menée depuis début 2014 sur 23 patients pluri-tronculaires connus. Les patients ont été recrutés dans le service de médecine nucléaire où ils étaient adressés pour une imagerie de perfusion de contrôle dans les 6 semaines après une coronarographie avec geste de revascularisation. Le protocole consistait en deux acquisitions dynamiques de 6 minutes, la première au repos après injection de $5 \mathrm{mCi}$ de ${ }^{99} \mathrm{Tc}$-tetrofosmine (185 MBq), et la seconde à 30 minutes après stress pharmacologique au dipyridamole et injection de $15 \mathrm{mCi}(555 \mathrm{MBq})$ de ${ }^{99} \mathrm{Tc}-$ tetrofosmine. Les acquisitions étaient démarrées concomitamment à l'injection du traceur de manière à capturer l'entrée vasculaire. L'analyse quantitative permettait l'obtention d'un index de réserve de perfusion myocardique (RPM) défini comme le ratio des débits de stress et de repos, à la fois global (sur l'ensemble du myocarde) et régional (par territoire coronaire). Tous les patients ont bénéficié dans les 15 jours après la scintigraphie d'une coronarographie de contrôle, utilisée comme examen de référence, permettant de définir pour chaque vaisseau coronaire son degré de sténose maximale. Un vaisseau était considéré comme obstrué en cas de sténose maximale supérieure à $50 \%$. Un sous-groupe de 12 patients (26 vaisseaux) a par ailleurs bénéficié de mesures invasives de réserve de perfusion (fractional flow reserve [FFR]).

À titre indicatif, la Fig. 5 montre un exemple de coupes reconstruites sur des acquisitions dynamiques de stress avec un échantillonnage temporel très fin (3 secondes) durant la phase vasculaire et plus grossier (30 secondes) durant la captation myocardique. Les résultats préliminaires sont encourageants et 


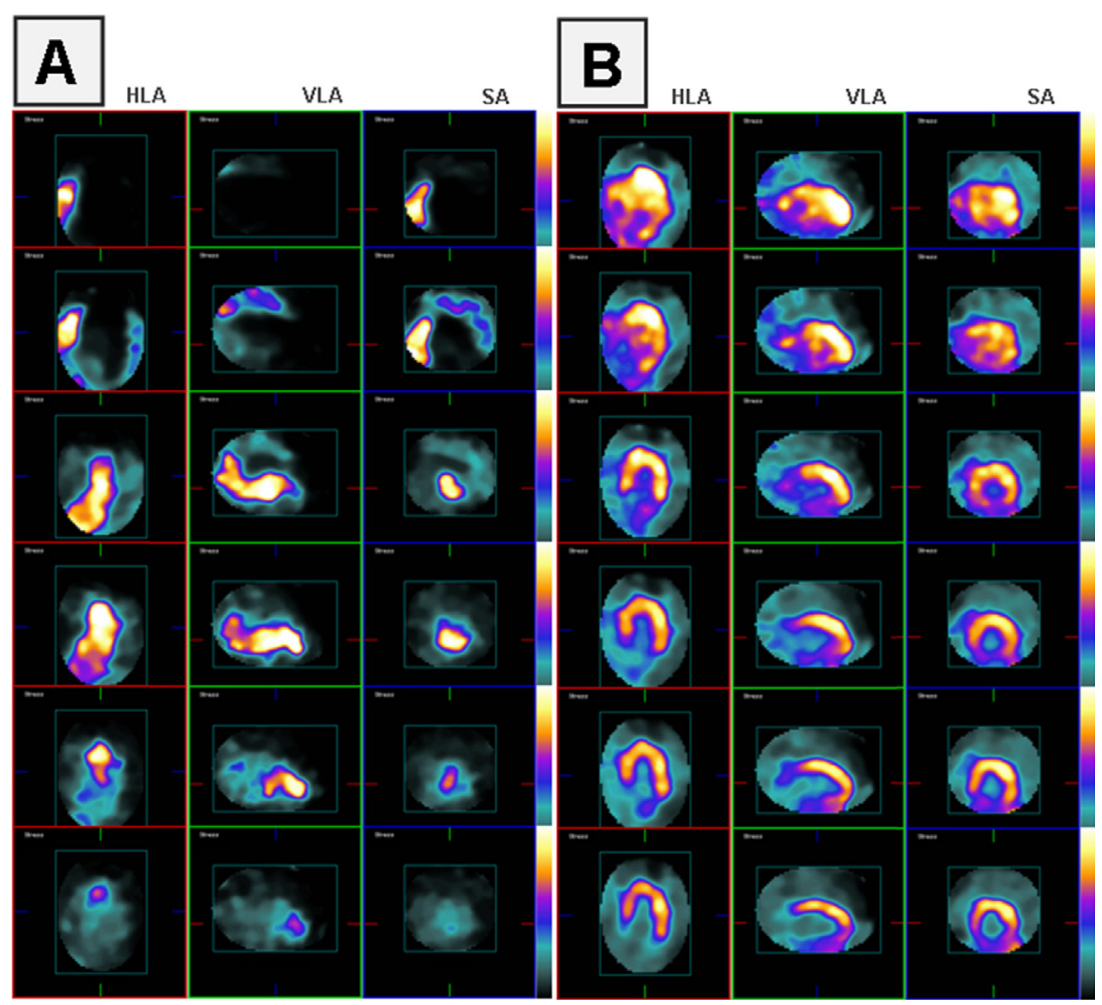

Fig. 5. Images dynamiques (A) de $3^{\prime}$ durant la phase d'entrée vasculaire et (B) de $30^{\prime}$ pendant la phase de captation myocardique. A. 3' dynamic images during vascular first-pass. B. 30' dynamic images during myocardial uptake.

indiquent une corrélation significative entre l'index RPM global et le nombre de vaisseaux obstrués. Au-delà, au niveau régional, il existe un lien très significatif entre les indices RPM régionaux et les constatations angiographiques en termes de degré de sténose maximale et en termes de FFR.

\section{Tomographie cavitaire}

La ventriculographie isotopique s'appuie sur un ensemble de techniques validées visant à évaluer les fonctions ventriculaires gauche et droite en termes de fraction d'éjection et de volumes télésystolique et télédiastolique. Elle est indiquée dans l'évaluation initiale et le suivi de l'insuffisance cardiaque, dans le cadre de la surveillance de traitements cardiotoxiques [32] et a montré son intérêt dans le diagnostic de cardiopathies plus rares [33]. Bien que la mesure de la fraction d'éjection du ventricule gauche (FEVG) sur acquisition planaire reste la référence du fait de sa grande reproductibilité, l'analyse des reconstructions tomographiques permet d'accéder à des mesures plus exactes de FEVG, d'évaluer la fonction systolique ventriculaire droite, les volumes ventriculaires et de mieux apprécier la cinétique segmentaire VD et VG. Ces données sont essentielles, en particulier pour les patients éligibles à une resynchronisation ventriculaire [34,35]. Une étude récente a démontré la faisabilité de mesures de FEVG sur des données tomographiques issues d'une caméra CZT [36]. Les résultats indiquent que la variabilité intra- et inter-observateur de ces mesures est meilleure que celle obtenue sur gamma-caméra d'Anger (à la fois en mode planaire et en tomographie).
Depuis 2009, un outil logiciel de traitement de données de tomo-ventriculographie isotopique a été mis au point au $\mathrm{CHU}$ de Montpellier sur la base de travaux de recherche menés depuis une quinzaine d'années dans le service de médecine nucléaire relatifs à la segmentation automatique des cavités cardiaques et à la modélisation des courbes de vidange ventriculaire [37,38]. L'outil s'appelle TomPool, fonctionne sous Windows, est entièrement gratuit et librement téléchargeable en ligne (http://scinti.edu.umontpellier.fr/recherche/ logiciels-a-telecharger/). Il supporte les fichiers dicom issus de tout type de gamma-caméra pourvu que les coupes aient préalablement été reconstruites et réorientées en vue HLA. Les premières étapes requièrent la définition du plan du septum, du plan des valves et du plan de l'infundibulum pulmonaire en tant que limite supérieure du ventricule droit. Un algorithme d'immersion en 4D est ensuite employé pour segmenter de manière totalement automatique les cavités cardiaques. Des outils de segmentation semi-automatique voire manuelle sont disponibles pour affiner la segmentation si nécessaire. Un des atouts du logiciel tient à la manière de modéliser les courbes de vidange ventriculaire obtenues à l'issue de la segmentation. L'approche classique consiste à appliquer un fit harmonique. Le fit mono-harmonique est connu pour induire un biais et sousestimer les mesures de fraction d'éjection. Il est par ailleurs mal adapté à la description d'un cycle cardiaque constitué d'une systole et d'une diastole de durées inégales et variables [39]. Le fit multi-harmonique, plus flexible, est aussi moins robuste et plus sensible au bruit statistique. L'approche originale employée dans TomPool repose sur la déformation d'une 
courbe de vidange de référence issue d'une population de patients sains [38]. Cette courbe subit des déformations selon l'axe des abscisses (temps) et des ordonnées (volume) qui sont optimisées de manière à modéliser au mieux les données bruitées. La grande flexibilité de la méthode lui confère une meilleure précision que le fit mono-harmonique. D'autre part, les déformations appliquées conservant les contraintes physiologiques applicables à une courbe de vidange ventriculaire, la robustesse est meilleure qu'avec un fit multi-harmonique. En sus des paramètres fonctionnels globaux (volumes, débit, fraction d'éjection, temps de fin de systole droit et gauche), le logiciel propose une estimation des paramètres fonctionnels locaux et segmentaires. Pour cela, il réalise une paramétrisation automatique des surfaces ventriculaires à partir de laquelle sont définis des éléments de volume élémentaires sur lesquels sont modélisées des courbes de vidange locales. Les paramètres fonctionnels calculés sont présentés à la fois sur un rendu volumique, sous forme d'histogramme et sur une vue polaire permettant une analyse affinée en termes de zone d'hypokinésie ou de dyskinésie (Fig. 6).

TomPool a été validé cliniquement par deux équipes indépendantes [40-42]. Les résultats obtenus par tomoventriculographie étaient dans les deux cas comparés à ceux de l'IRM cardiaque considérée comme gold standard. Les deux études ont démontré une excellente corrélation $(p<0,001)$ entre les deux méthodes en ce qui concerne les volumes ventriculaires et les fractions d'éjection systolique, tant du ventricule gauche que du ventricule droit. La publication récente de l'équipe toulousaine a aussi étudié la reproductibilité inter-observateur des résultats de TomPool qui s'est avérée très satisfaisante (respectivement $94 \%$ et $90 \%$ pour la fraction d'éjection et les volumes du VG, $81 \%$ et $77 \%$ pour la fraction d'éjection et les volumes du VD) avec des résultats légèrement moins bons pour les paramètres ventriculaires droits à rapporter à la variabilité liée au choix par l'opérateur de la limite supérieure du VD.

\section{Scintigraphie à la MIBG}

Analogue structural de la guanéthidine, agent bloquant adrénergique, la MIBG a la même structure que la noradrénaline. Elles partagent toutes deux les mêmes mécanismes de stockage et de captation au niveau des terminaisons nerveuses sympathiques. La MIBG a été largement décrite pour être un indicateur fiable de l'intégrité du tonus sympathique cardiaque [43]. Ce tonus peut être altéré en particulier dans l'insuffisance cardiaque. Dans cette pathologie chronique, la tomoscintigraphie (TEMP) à la ${ }^{123}$ I-MIBG joue depuis plusieurs années un rôle diagnostique et pronostique croissant. Elle permet d'identifier les sujets à risque majeur de trouble du rythme
VD
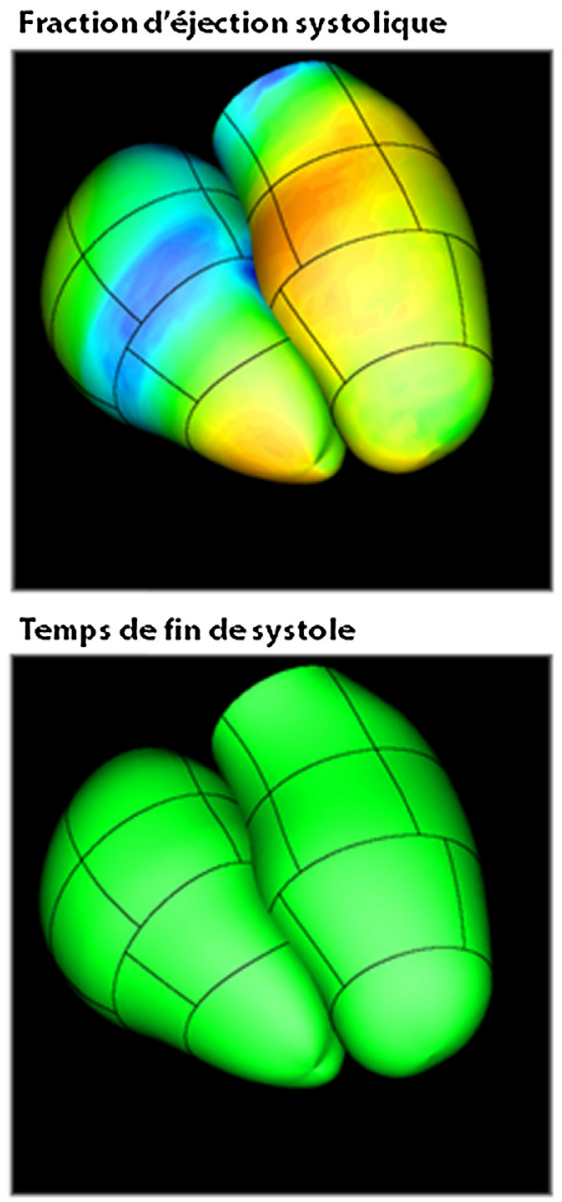

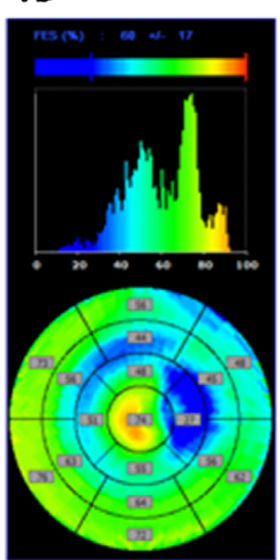

VG

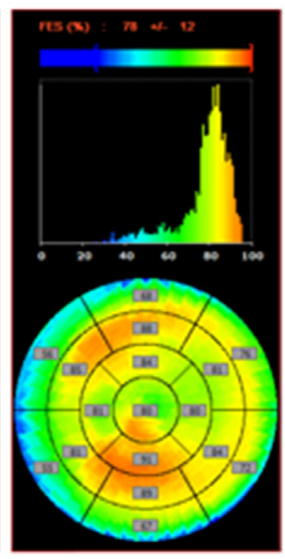

VD

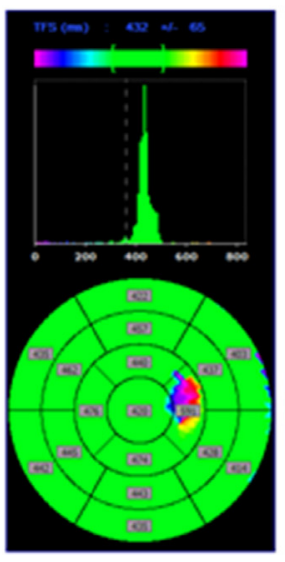

VG

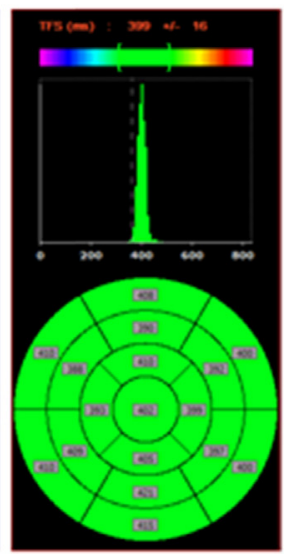

Fig. 6. Logiciel TomPool développé au CHU de Montpellier. Exemple de présentation des résultats relatifs à la cinétique locale et segmentaire. TomPool software developed in Montpellier University Hospital. 
pouvant bénéficier d'un défibrillateur implantable en prévention primaire [44-46]. Encore trop peu utilisée par nos confrères cardiologues, car non intégré dans leurs recommandations, cet outil de cardiologie nucléaire permettrait la stratification personnalisée du risque rythmique. En effet, les morts subites par cause cardiaque représentent encore une part importante des décès dans les pays industrialisés.

À l'instar de l'insuffisance cardiaque, la pathologie coronaire (syndromes coronariens aigus) est responsable de troubles du rythme précoces liés à l'infarctus du myocarde qui sont bien connus et prévenus à la phase aiguë. En revanche, le risque de trouble du rythme après reperfusion est moins bien évalué.

Après un infarctus du myocarde, la dénervation sympathique régionale dépasse la zone du défect perfusionnel (plus grande sensibilité du tissu nerveux à l'hypoxie) [47]. C'est ici que le concept de zone gâchette apparaît. Il s'agit d'un territoire myocardique bien vascularisé ( $>50 \%$ du maximum de fixation en $\left.{ }^{201} \mathrm{Tl}\right)$, mais mal innervé sur le plan sympathique $(<50 \% \mathrm{du}$ maximum de fixation en $\left.{ }^{123} \mathrm{I}-\mathrm{MIBG}\right)$. Vraisemblablement proarythmogènes [48], ces zones gâchettes peuvent être identifiées en TEMP myocardique double isotope traceur perfusionnel $/{ }^{123} \mathrm{I}$-MIBG. À l'ère des caméras CZT, ces protocoles double isotope permettent un gain de temps et de confort pour le patient ainsi que l'optimisation de l'occupation des installations.

Dans la plupart des travaux s'intéressant à cette problématique, les auteurs utilisent des protocoles double isotope ${ }^{99} \mathrm{~m} \mathrm{Tc} /{ }^{123} \mathrm{I}-\mathrm{MIBG}$ séquentiels ou simultanés [44,49-51]. L'utilisation de protocole ${ }^{201} \mathrm{Tl} /{ }^{123} \mathrm{I}-\mathrm{MIBG}$ pour l'identification de zone gâchette nécessite une limitation optimale de la détection du diffusé Compton de $1{ }^{123}$ I dans le pic photo-électrique du ${ }^{201} \mathrm{Tl}$. En effet, une diminution du contraste dans les images de thallium mène à une surestimation de la viabilité myocardique et donc de l'étendue des zones gâchettes. Malgré leur résolution en énergie accrue, la nécessité d'une correction du diffusé avec les caméras CZT reste incertaine pour ce type de protocole.

Au cours d'un travail mené en collaboration avec l'équipe de cardiologie du CHU de Montpellier, nous nous sommes intéressés à l'élaboration d'un protocole TEMP applicable en routine clinique pour évaluer l'incidence des zones gâchettes dans une population de patients pris en charge pour syndrome coronarien aigu ST+ (SCA ST+) après revascularisation (Fig. 7).

Nous avons en premier lieu étudié l'intérêt d'une correction du diffusé puis la nécessité d'une acquisition précoce ${ }^{123} \mathrm{I}$ MIBG pour l'étude de l'innervation sympathique cardiaque.

$\mathrm{Au}$ cours de leur hospitalisation et après reperfusion de l'ensemble des lésions coronaires causales du SCA ST+,
3 TEMP étaient réalisées selon le protocole présenté sur la Fig. 7 : une acquisition simple isotope thallium (perfusion de repos $\left[\mathrm{Tl}_{1}\right]$ ), deux acquisitions double isotope (MIBG précoce $\left(\mathrm{MIBG}_{1}\right) /$ perfusion de repos $\left(\mathrm{Tl}_{2}\right)$ et $\mathrm{MIBG}$ tardive $\left(\mathrm{MIBG}_{3}\right) /$ viabilité $\left.\left[\mathrm{Tl}_{3}\right]\right)$. Les acquisitions étaient réalisées sur caméra Discovery NM530c (GE Healthcare) avec les paramètres suivants : mode liste, matrice $32 \times 32$, taille de pixel $4 \mathrm{~mm}$, fenêtres énergétiques centrées sur $67 \mathrm{keV} \pm 10 \%$ et $159 \mathrm{keV} \pm 10 \%$. Les projections étaient ensuite reconstruites (MAP-OSEM, 30 itérations, $\alpha=\beta=0,4$, post-filtre Butterworth 10/0,4) sur station Xeleris sans correction du diffusé pour toutes les acquisitions et avec correction du diffusé par la méthode des trois fenêtres énergétiques (triple energy window [TEW]) pour celle du thallium tardif. Le but étant de limiter la détection du diffusé Compton de $1{ }^{123}$ I dans la fenêtre photoélectrique du ${ }^{201} \mathrm{Tl}$. La méthode TEW utilisée s'appuyait sur les travaux de plusieurs équipes japonaises [52-54]. Les fenêtres énergétiques choisies étaient les suivantes : $70 \mathrm{keV} \pm 12 \%$ pour la fenêtre principale $(\mathrm{W}=8,4 \mathrm{keV})$, et $60,1 \mathrm{keV} \pm 2 \%$ $\left(\mathrm{w}_{1}=1,2 \mathrm{keV}\right)$ et $79,9 \mathrm{keV} \pm 2 \%\left(\mathrm{w}_{2}=1,6 \mathrm{keV}\right)$ pour les deux fenêtres latérales. L'estimation des projections corrigées du diffusé $\left(\mathrm{Tl}_{3 \mathrm{c}}\right)$ était obtenue selon la formule suivante [53] où $\mathrm{Tl}^{1}$ et $\mathrm{Tl}^{\mathrm{k}}$ représentent le nombre de photons dans les fenêtres latérales :

$$
\mathrm{Tl}_{3 \mathrm{c}}=\mathrm{Tl}_{3}-\frac{\mathrm{W}}{\mathrm{w}_{1}+\mathrm{w}_{2}}\left(\mathrm{Tl}^{\mathrm{l}}+\mathrm{Tl}^{\mathrm{h}}\right)=\mathrm{Tl}_{3}-3 \cdot\left(\mathrm{Tl}^{\mathrm{l}}+\mathrm{Tl}^{\mathrm{h}}\right)
$$

Pour l'analyse de chaque acquisition sur QPS/QGS ${ }^{\circledR}$ (Cedars Sinaï), le ventricule gauche était divisé en 17 segments en représentation polaire et un score de fixation (échelle de 0 : fixation $\geq 70 \%$ à 5 : fixation $<10 \%$ ) était attribué à chaque segment $[10,55]$. En sommant chaque score des 17 segments, pour chaque patient, étaient ainsi calculés des scores sommés (SS) de perfusion et d'innervation ( $\mathrm{SS} \mathrm{Tl}_{1}, \mathrm{SS} \mathrm{Tl}_{2}, \mathrm{SS} \mathrm{Tl}_{3}, \mathrm{SS}$ $\mathrm{Tl}_{3 \mathrm{c}}$, SS $\mathrm{MIBG}_{1}$, SS MIBG 3 ). De même des scores sommés gâchette $\left(\mathrm{SSG}_{1}, \mathrm{SSG}_{3}, \mathrm{SSG}_{3 \mathrm{c}}\right)$ étaient définis en considérant pour les calculer l'acquisition tardive $\mathrm{MIBG}_{3}$ et, respectivement, l'acquisition $\mathrm{Tl}_{1}, \mathrm{Tl}_{3}$ et $\mathrm{Tl}_{3 \mathrm{c}}$.

En parallèle, les patients réalisaient une IRM myocardique et/ou une ETT afin d'évaluer l'étendue de la zone de nécrose liée à l'infarctus récent. Ces examens étaient considérés comme gold standard pour comparer les trois acquisitions thallium entre elles $\left(\mathrm{Tl}_{1}, \mathrm{Tl}_{3}, \mathrm{Tl}_{3 \mathrm{c}}\right)$ afin de déterminer laquelle présentait les meilleures valeurs diagnostiques pour évaluer les nécroses myocardiques.

Sans correction du diffusé, le diffusé de $1{ }^{123}$ I induisait des différences significatives entre les $\mathrm{SS} \mathrm{Tl}_{1}$ vs $\mathrm{Tl}_{2}$ et $\mathrm{Tl}_{3}$. Les $\mathrm{SS}$ MIBG étaient significativement plus importants sur les

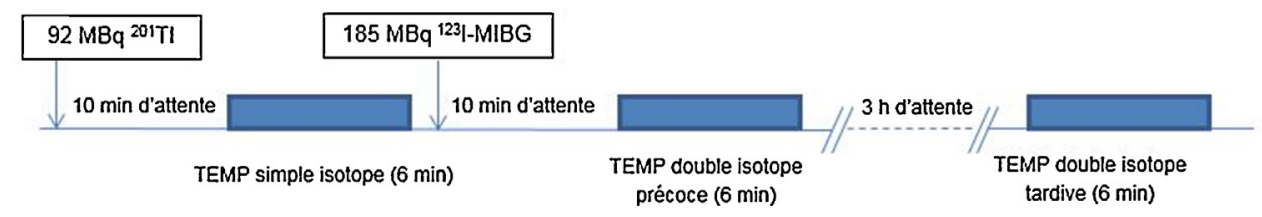

Fig. 7. Protocole d'acquisition double isotope ${ }^{201} \mathrm{Tl} /{ }^{123} \mathrm{I}-\mathrm{MIBG}$ pour l'étude des zones gâchettes.

${ }^{201} \mathrm{Tl}^{123} \mathrm{I}-\mathrm{MIBG}$ double isotope acquisition protocol. 

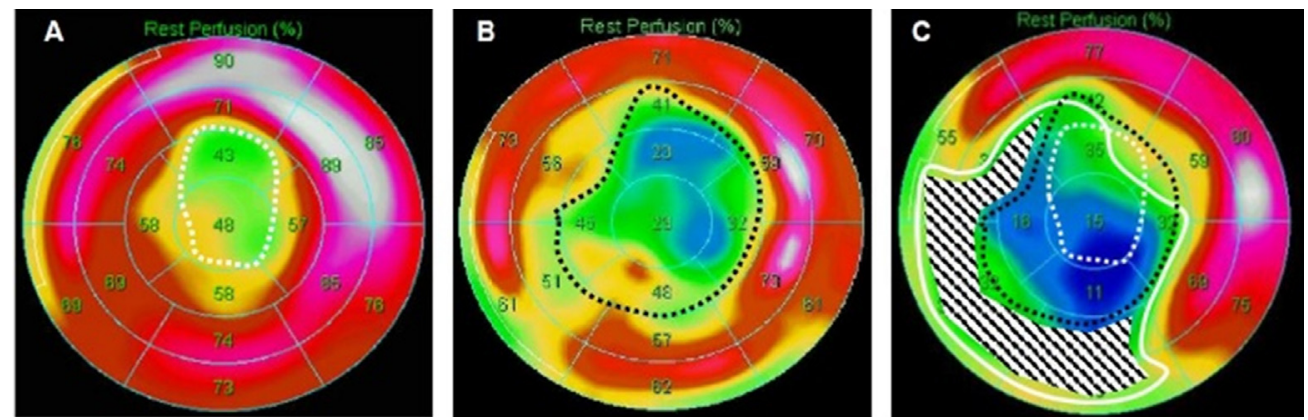

Fig. 8. Exemple d'acquisition $\mathrm{Tl}_{3}(\mathrm{~A}), \mathrm{Tl}_{3 \mathrm{c}}(\mathrm{B})$ et $\mathrm{MIBG}_{3}(\mathrm{C})$. La zone hachurée représente l'étendue de la zone gâchette (myocarde viable mais dénervé). On visualise aisément que l'étendue de celle-ci varie en considérant tantôt l'acquisition $\mathrm{Tl}_{3}$ (pointillés blanc), tantôt l'acquisition $\mathrm{Tl}_{3 \mathrm{c}}$ (pointillés noir).

Example of $(A) T l_{3},(B) T l_{3 c}$, and $(C) M I B G_{3}$ acquisitions. The hatched area represents the extent of the trigger zone (viable but denervated myocardium). This extent is clearly dependent upon the chosen $\mathrm{Tl}$ images: $\mathrm{Tl}_{3}$ (white dotted line) or $\mathrm{Tl}_{3 c}$ (black dotted line).

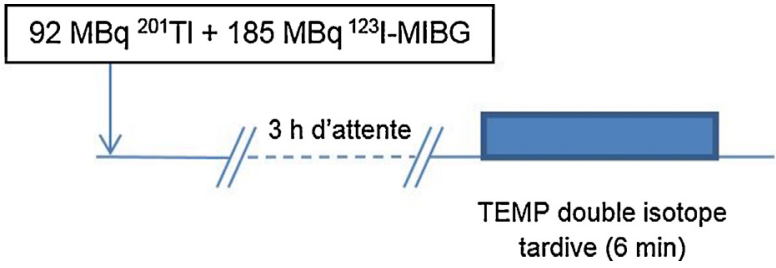

Fig. 9. Protocole optimisé double isotope simultané pour exploration des zones gâchettes.

Optimized double isotope protocol for the detection of trigger zones.

acquisitions tardives $\left(\mathrm{MIBG}_{3}\right)$ et les SSG étaient significativement plus importants considérant les acquisitions $\mathrm{MIBG}_{3}$ et $\mathrm{Tl}_{3}$. Avec correction du diffusé, les $\mathrm{SS} \mathrm{Tl}_{3 c}$ étaient statistiquement plus importants que les SS des acquisitions non corrigées du diffusé. Ainsi les $\mathrm{SSG}_{3 \mathrm{c}}$ étaient significativement inférieurs aux $\mathrm{SSG}_{1}$ et $\mathrm{SSG}_{3}$ (Fig. 8).

Comparées aux acquisitions thallium sans correction du diffusé et considérant l'IRM et/ou l'ETT comme gold standard, les acquisitions $\mathrm{Tl}_{3 \mathrm{c}}$ présentaient les meilleurs couples de valeurs diagnostiques (Se $72 \%$, Sp $91 \%$, VPP $78 \%$, VPN $88 \%$ vs $\operatorname{Se} 52 \%$, Sp $92 \%$, VPP $75 \%$, VPN $82 \%$ pour $\mathrm{Tl}_{1}$ et $\mathrm{Se} 37 \%$, Sp $95 \%$, VPP $77 \%$, VPN $77 \%$ pour $\mathrm{Tl}_{3}$ ). Ainsi, malgré une résolution en énergie accrue, une correction du diffusé est donc nécessaire pour ce protocole double isotope simultané ${ }^{201} \mathrm{Tl} /{ }^{123} \mathrm{I}$-MIBG sur CZT dans l'imagerie des zones gâchettes. Par ailleurs, seule l'acquisition MIBG tardive permettait une évaluation correcte de l'innervation sympathique. Considérant nos résultats, nous pouvons proposer un protocole optimisé (Fig. 9) pour ce type d'indication qui se résume à un temps patient dans le service d'environ 20 min (accueil + injection + acquisition) et un temps d'occupation caméra de $10 \mathrm{~min}$ (installation du patient +6 min d'acquisition).

\section{Conclusion}

L'avènement des caméras à semi-conducteurs représente sans conteste l'évolution technologique majeure de ces dernières années dans le monde de la cardiologie isotopique. L'optimisation des protocoles de routine en perfusion myocardique et en tomographie cavitaire permet d'accroître le confort des patients et de réduire les doses reçues tout en satisfaisant aux exigences de rentabilité. Les résultats préliminaires des travaux de recherche menés au CHU de Montpellier tendent à confirmer la pertinence de mesures quantitatives de réserve coronaire par SPECT dynamique et à montrer la faisabilité d'une détection précoce du risque rythmique en post-SCA par acquisition double isotope ${ }^{201} \mathrm{Tl} /{ }^{123} \mathrm{I}-\mathrm{MIBG}$ avec correction du diffusé Compton.

\section{Déclaration d'intérêts}

Les auteurs déclarent ne pas avoir de conflits d'intérêts en relation avec cet article.

\section{Références}

[1] Madsen MT. Recent advances in SPECT imaging. J Nucl Med 2007;48(4)661-73.

[2] Gordon DePuey E. Advances in cardiac processing software. Semin Nucl Med 2014;44(4)252-73.

[3] Case JA, Bateman TM. Taking the perfect nuclear image: quality control acquisition, and processing techniques for cardiac SPECT, PET, and hybrid imaging. J Nucl Cardiol 2013;20(5)891-907.

[4] Slomka PJ, Berman DS, Germano G. New cardiac cameras: single-photon emission CT and PET. Semin Nucl Med 2014;44(4)232-51.

[5] Garcia EV, Faber TL, Esteves FP. Cardiac dedicated ultrafast SPECT cameras: new designs and clinical implications. J Nucl Med 2011;52(2)210-7.

[6] Aarsvold JN, Wernick MN. Emission tomography: the basics of PET and SPECT. Academic Press; 2004

[7] Takahashi Y, et al. Performance of a semiconductor SPECT system: comparison with a conventional Anger-type SPECT instrument. Ann Nucl Med 2013;27(1)11-6.

[8] Duvall WL, et al. Reduced isotope dose and imaging time with a highefficiency CZT SPECT camera. J Nucl Cardiol 2011;18(5)847-57.

[9] Salerno M, Beller GA. Noninvasive assessment of myocardial perfusion. Circ Cardiovasc Imaging 2009;2(5)412-24.

[10] Hesse B, et al. EANM/ESC procedural guidelines for myocardial perfusion imaging in nuclear cardiology. Eur J Nucl Med Mol Imaging 2005;32(7)855-97.

[11] Burrell S, MacDonald A. Artifacts and pitfalls in myocardial perfusion imaging. J Nucl Med Technol 2006;34(4)193-211.

[12] Hage FG, et al. The impact of viability assessment using myocardial perfusion imaging on patient management and outcome. J Nucl Cardiol 2010;17(3)378-89. 
[13] Al Moudi M, Sun ZH. Diagnostic value of (18)F-FDG PET in the assessment of myocardial viability in coronary artery disease: a comparative study with $(99 \mathrm{~m}) \mathrm{Tc}$ SPECT and echocardiography. J Geriatr Cardiol 2014;11(3)229-36

[14] Imbert L, et al. Compared performance of high-sensitivity cameras dedicated to myocardial perfusion SPECT: a comprehensive analysis of phantom and human images. J Nucl Med 2012;53(12)1897-903.

[15] Cochet $\mathrm{H}$, et al. Absolute quantification of left ventricular global and regional function at nuclear MPI using ultrafast CZT SPECT: initial validation versus cardiac MR. J Nucl Med 2013;54(4)556-63.

[16] Tanaka H, et al. Diagnostic performance of a novel cadmium-zinctelluride gamma camera system assessed using fractional flow reserve. Circ J 2014;78(11)2727-34

[17] Mouden $M$, et al. Impact of a new ultrafast CZT SPECT camera for myocardial perfusion imaging: fewer equivocal results and lower radiation dose. Eur J Nucl Med Mol Imaging 2012;39(6)1048-55.

[18] Verger A, et al. Comparison between stress myocardial perfusion SPECT recorded with cadmium-zinc-telluride and Anger cameras in various study protocols. Eur J Nucl Med Mol Imaging 2013;40(3)331-40.

[19] Fiechter M, et al. Nuclear myocardial perfusion imaging with a novel cadmium-zinc-telluride detector SPECT/CT device: first validation versus invasive coronary angiography. Eur J Nucl Med Mol Imaging 2011; 38(11)2025-30.

[20] Gimelli A, et al. High diagnostic accuracy of low-dose gated-SPECT with solid-state ultrafast detectors: preliminary clinical results. Eur J Nucl Med Mol Imaging 2012;39(1)83-90.

[21] Husain SS. Myocardial perfusion imaging protocols: is there an ideal protocol? J Nucl Med Technol 2007;35(1)39.

[22] Herzog BA, et al. Nuclear myocardial perfusion imaging with a cadmiumzinc-telluride detector technique: optimized protocol for scan time reduction. J Nucl Med 2010;51(1)46-51.

[23] Buechel RR, et al. Ultrafast nuclear myocardial perfusion imaging on a new gamma camera with semiconductor detector technique: first clinical validation. Eur J Nucl Med Mol Imaging 2010;37(4)773-8.

[24] Ghatk A, et al. Role of imaging for acute chest pain syndromes. Semin Nucl Med 2013;43(2)71-81.

[25] Garcia EV. Are SPECT measurements of myocardial blood flow and flow reserve ready for clinical use? Eur J Nucl Med Mol Imaging 2014; 41(12)2291-3.

[26] Ziadi. et al. Does quantification of myocardial flow reserve using rubidium-82 positron emission tomography facilitate detection of multivessel coronary artery disease? J Nucl Cardiol 2012;19(4)670-80.

[27] Murthy. et al. Improved cardiac risk assessment with noninvasive measures of coronary flow reserve. Circulation 2011;124(20)2215-24.

[28] Ben-Haim. et al. JNM 2013. Quantification of myocardial perfusion reserve using dynamic SPECT imaging in humans: a feasibility study. J Nucl Med 2013;54(6)873-9.

[29] Bailing H, et al. Quantitation of myocardial blood flow and myocardial flow reserve with $99 \mathrm{mTc}$-sestamibi dynamic SPECT/CT to enhance detection of coronary artery disease. Eur J Nucl Med Mol Imaging 2014:41:2294-300.

[30] Klein R, Beanlands RS, deKemp RA. Quantification of myocardial blood flow and flow reserve: Technical aspects. J Nucl Cardiol 2010; 17(4)555-70.

[31] Schelbert HR. Anatomy and physiology of coronary blood flow. J Nucl Cardiol 2010;17(4)545-54

[32] Hesse B, et al. EANM/ESC guidelines for radionuclide imaging of cardiac function. Eur J Nucl Med Mol Imaging 2008;35(4)851-85.

[33] Mariano-Goulart D, et al. Diagnosis of diffuse and localized arrhythmogenic right ventricular dysplasia by gated blood-pool SPECT. J Nucl Med 2007;48(9)1416-23.

[34] Lalonde M, et al. SPECT gated blood pool phase analysis of lateral wall motion for prediction of CRT response. Int $\mathrm{J}$ Cardiovasc Imaging 2014;30(3)559-69.
[35] Lalonde M, et al. Development and optimization of SPECT gated blood pool cluster analysis for the prediction of CRT outcome. Med Phys 2014;41(7)072506

[36] Jensen MM, Schmidt U, Huang C, Zerahn B. Gated tomographic radionuclide angiography using cadmium-zinc-telluride detector gamma camera; comparison to traditional gamma cameras. J Nucl Cardiol 2014;21(2)384-96

[37] Mariano-Goulart D, et al. Semi-automatic segmentation of gated blood pool emission tomographic images by watersheds:application to the determination of right and left ejection fractions. Eur J Nucl Med 1998;25(9)1300-7.

[38] Caderas de Kerleau C, et al. Automatic generation of noise-free timeactivity curve with gated blood-pool emission tomography using deformation of a reference curve. IEEE Trans Med Imaging 2004;23(4)485-91.

[39] Hansen CL. Limitations of parametric modeling of the left ventricle using first harmonic analysis: possible role for gaussian modeling. J Nucl Cardiol 2014;21(4)723-9.

[40] Sibille. et al. Comparative values of gated blood-pool SPECT and CMR for ejection fraction and volume estimation. Nucl Med Commun 2011;32(2)121-8.

[41] Sibille. et al. Influence of CT-based attenuation correction in assessment of left and right ventricular functions with count-based gated blood-pool SPECT. J Nucl Cardiol 2011;18(4)642-9.

[42] Dercle. et al. Is Tompool accurate to diagnose right and left ventricular dysfunction in a clinical setting? J Nucl Cardiol 2014;21(5)1011-22.

[43] Flotats A, Carrio I. Cardiac neurotransmission SPECT imaging. J Nucl Cardiol 2004;11(5)587-602.

[44] Boogers MJ, et al. Cardiac sympathetic denervation assessed with 123iodine metaiodobenzylguanidine imaging predicts ventricular arrhythmias in implantable cardioverter-defibrillator patients. J Am Coll Cardiol 2010;55(24)2769-70

[45] Merlet P, et al. Prognostic value of cardiac metaiodobenzylguanidine imaging in patients with heart failure. J Nucl Med 1992;33(4)471-7.

[46] Agostini D, et al. Cardiac 123I-MIBG scintigraphy in heart failure. Q J Nucl Med Mol Imaging 2008;52(4)369-77.

[47] Matsunari I, et al. Extent of cardiac sympathetic neuronal damage is determined by the area of ischemia in patients with acute coronary syndromes. Circulation 2000;101(22)2579-80.

[48] Klein T, et al. Three-dimensional 123I-meta-iodobenzylguanidine cardiac innervation maps to assess substrate and successful ablation sites for ventricular tachycardia: feasibility study for a novel paradigm of innervation imaging. Circ Arrythm Electrophysiol 2015;8(3)583-91.

[49] Gimelli A, et al. Regional heterogeneity in cardiac sympathetic innervation in acute myocardial infarction: relationship with myocardial oedema on magnetic resonance. Eur J Nucl Med Mol Imaging 2014;41(9)1692-700.

[50] Podrid PJ, et al. Role of the sympathetic nervous system in the genesis of ventricular arrhythmia. Circulation 1990;82 suppl. 1(2)I103-13.

[51] Du Y, et al. Simultaneous Tc-99m/I-123 dual-radionuclide myocardial perfusion/innervation imaging using Siemens IQ-SPECT with SMARTZOOM collimator. Phys Med Biol 2014;59(11)2813-20.

[52] Ogawa K, et al. A practical method for position-dependent Comptonscatter correction in single photon emission CT. IEEE Trans Med Imaging 1991;10(3)408-12.

[53] Ichihara T, et al. Compton scatter compensation using the triple-energy window method for single- and dual-isotope SPECT. J Nucl Med 1993; 34(12)2216-20.

[54] Yang JT, et al. Clinical value of triple-energy window scatter correction in simultaneous dual-isotope single-photon emission tomography with 123IBMIPP and 201Tl. Eur J Nucl Med 1997;24(9)1099-100.

[55] Cerqueira MD, et al. Standardized myocardial segmentation and nomenclature for tomographic imaging of the heart. A statement for healthcare professionals from the Cardiac Imaging Committee of the Council on Clinical Cardiology of the American Heart Association. Int J Cardiovasc Imaging 2002;18(1)539-42. 